\section{Bamboo flowering and pandas}

SiR-The flowering and subsequent natural dieback of arrow bamboo in Wolong and other areas in China in 1983 did not cause the mass starvation of pandas that was feared because they foraged on alternative bamboo species or unflowered arrow bamboo patches ${ }^{1-3}$. Now that the perceived crisis has passed, efforts to conserve the panda can focus on the urgent problems of poaching, habitat preservation, release of captive animals and development of a captive-breeding programme $e^{1.4,5}$. But an unusual opportunity to restore degraded panda habitat should receive equal attention.

Even though bamboo density is greater in those areas of Wolong that were clearfelled in the 1960s and 1970s than in the still-forested areas, pandas prefer forest habitat. Few trees, especially conifers, became re-established in the clearcuts because of the dense vegetatively propogated bamboo, but conifers dominate the subalpine forest in Wolong and old hollow conifers serve as maternity dens for pandas ${ }^{5-7}$.

We therefore urge the immediate planting of native conifers in the areas cleared by the 1983 dieback before the regrowing arrow bamboo has reached a size that would inhibit conifer establishment - this requires $10-15$ years. Rapid development of conifer forest from planted seedlings may also help reduce the high bamboo seedling mortality observed in clearcuts ${ }^{8}$. Cost-effective opportunities to restore degraded panda habitat to conifer forest are infrequent because arrow bamboo will not flower again for 45-50 years ${ }^{5.9}$.

\section{Department of Geography,}

University of Maryland Baltimore County,

Baltimore, Maryland 21228, USA

Department of Biology,

Nanchong Teacher's College,

Nanchong, Sichuan,

People's Republic of China

1. Schaller, G. B. Nature 327, 562 (1987).

2. Taylor, A. H. \& Qin Zisheng J. appl. Ecol. 24, 419-433 (1987).

3. Johnson, K. G., Schaller, G. B. \& Hu Jinchu Nat. geog. Res. 4, 161-177 (1988)

4. O'Brien, S. J. \& Knight, J. A. Nature 325, 758-759 (1987).

5: Schaller, G. B., Hu Jinchu, Pan Wenshi \& Zhu Jing The Giant Pandas of Wolong xiii, 298 (University of Chicago Press, 1985).

6. Taylor, A. H. \& Qin Zisheng J. Ecol. 76, 1204-1218 (1988).

7. Taylor, A. H. \& Qin Zisheng Biol. Consen, 47, 83-108 (1989).

8. Taylor, A. H. \& Qin Zisheng Am. J. Bot. 75, 1065-1073 (1988).

9. Campbell, J. J. N. \& Qin Zisheng J. Bamboo Soc. Am.4 1-34 (1983).

\title{
Basis of hepatitis delta virus disease?
}

SIR-Symons ${ }^{1}$ has recently discussed a reported $^{2}$ sequence complementarity between the six viroid 'species' that cause disease in tomato plants and tomato 7S RNA, a small cytoplasmic RNA present in various organisms as a component of the signal recognition particle, a structure involved in the translocation of secretory and membrane-associated proteins. It has been suggested that formation of hybrids between viroids and 7S RNA may be a

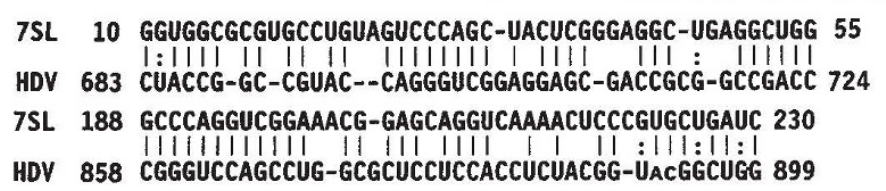

Proposed base-pairing between human 7SL RNA and anti-genomic HDV RNA. Colons indicate G:U base-pairs. Lowercase letters denote the two nucleotides that are identical in two reported HDV RNA sequences ${ }^{4,6}$ but differ in a third ${ }^{7}$.

factor in disease $e^{1,2}$. We wish to extend that suggestion to the hepatitis delta virus (HDV), a human pathogen that causes severe liver disease (delta hepatitis) throughout the world ${ }^{3}$.

Similarities between viroids and HDV have been demonstrated previously. Like viroids, HDV has a circular, single-stranded RNA genome that possesses a high degree of self-complementarity ${ }^{4}$ HDV RNA is believed to replicate via a rolling cycle mechanism through an anti-genomic
RNA replication intermediate 4 . Also, limited sequence similarity has been reported between viroids and HDV RNA ${ }^{4}$.

We have found that an extensive complementarity exists between two regions of human 7SL, RNA ${ }^{5}$ (the counterpart of tomato 7S RNA) and anti-genomic HDV $\mathrm{RNA}^{4}$ (see figure). The first region involves nucleotides $10-55$ of 7S RNA and 683-724 of the HDV replicative intermediate. In a 48-nucleotide domain, 35 $(73 \%)$ bases are complementary (including two $\mathrm{G}: \mathrm{U}$ base-pairs); three stretches of 6-8 nucleotides are perfectly base-paired. The second region of complementarity is found between bases 188-230 of 7SL RNA and 858-899 of antigenomic HDV RNA. In a 44-nucleotide domain, $34(77 \%)$ bases are complementary (including three $\mathrm{G}: \mathrm{U}$ base-pairs). Two long stretches, 12 and 9 bases in length, of perfectly paired nucleotides are found at the ends of this domain. The sequences that separate these two regions, although approximately of the same length (132 and 133 nucleotides), do not show any significant complementarity. Of the 69 nucleotides of the anti-genomic
HDV RNA complementary to 7SL RNA, 67 are invariant among the three known HDV sequences ${ }^{4.6 .7}$, whereas other regions of HDV show a rather high heterogeneity. Thus, the region of HDV anti-genome exhibiting complementarity with 7SL RNA is highly conserved among the known virus isolates.

The suggested base-pairing between human 7SL RNA and anti-genomic HDV RNA is even more extensive (and possibly more stable) than that between tomato $7 \mathrm{~S}$ RNA and the six viroids. Interestingly, the region in tomato 7S RNA (bases 2667) thought to be capable of annealing to viroid RNA corresponds to one of the regions of the human 7SL RNA complementary to the anti-genomic HDV RNA. However, due to sequence differences between tomato 7S RNA and its human counterpart ${ }^{2}$, there is no significant similarity between anti-genomic HDV RNA and viroid RNA in this region.

Thus, just as viroids may cause disease by base-pairing to a host structural RNA, so may a replicative intermediate of HDV. As HDV is known to replicate inside the nuclei of infected hepatocytes ${ }^{8}$, its anti-genomic form may anneal to the 7SL RNA shortly after the host RNA is transcribed and before it becomes incorporated in the signal recognition particle. This hypothesis is consistent with the notion that HDV is directly cytopathic ${ }^{3}$ and can be tested experimentally. If true, it might lead to new therapeutic strategies for the control of delta hepatitis.

FRANCESCO NEGRO JOHN L. GERIN

Division of Molecular Virology and Immunology,

Georgetown University,

Rockville, Maryland 20852, USA

ROBERT H. PURCELL

ROGER H. MILLER

Hepatitis Viruses Section,

National Institute of Allergy and

Infectious Diseases,

Bethesda, Maryland 20892, USA

1. Symons, B. Nature $336,542-543$ (1989).

2. Haas, B. et al. EMBO J. 7, 4063-4074 (1988)

3. Rizzetto, M. et al. (eds) Hepatitis Delta Virus and its Infection (Liss, New York, 1987)

4. Wang, W.S. et al. Nature 323, 508-514 (1986).

5. Ullu, E. \& Tschudi, C. Nature 312, 171-172 (1984)

6. Makino, S. et al. Nature 329, 343-346 (1987).

. Kuo, M.Y.P. et al. J. Virol. 62, 1855-1861 (1988).

8. Gowans, E.J. et al. Virology 167, 274-278 (1988)

\section{Aurorae aplenty}

SIR-Schröder's suggestion', that the Maunder minimum ${ }^{2}$ of solar activity does not show up in European auroral records, is corroborated by historical evidence from the Soviet Union. Although the great aurora of 16 March 1716 (Julian) seemed to awaken the scientific society of the time, initiating studies of the ionosphere, there were nevertheless useful observations made in the middle latitudes 